\title{
EBNA1 binding and epigenetic regulation of gastrokine tumor suppressor genes in gastric carcinoma cells
}

\author{
Fang Lu', Italo Tempera ${ }^{2}$, Hyunna T Lee ${ }^{1}$, Karen DeWispelaere ${ }^{1}$ and Paul M Lieberman ${ }^{1 *}$
}

\begin{abstract}
Background: Epstein-Barr Virus (EBV) latently infects $~ 10 \%$ of gastric carcinomas (GC). Epstein-Barr Nuclear Antigen 1 (EBNA1) is expressed in EBV-associated GC, and can bind host DNA, where it may impact cellular gene regulation. Here, we show that EBNA1 binds directly to DNA upstream of the divergently transcribed GC-specific tumor suppressor genes gastrokine 1 (GKN1) and gastrokine 2 (GKN2).

Methods: We use ChIP-Seq, ChIP-qPCR, and EMSA to demonstrate that EBNA1 binds directly to the GKN1 and GKN2 promoter locus. We generate AGS-EBV, and AGS-EBNA1 cell lines to study the effects of EBNA1 on GKN1 and GKN2 mRNA expression with or without 5' azacytidine treatment.

Results: We show that gastrokine genes are transcriptionally silenced by DNA methylation. We also show that latent EBV infection further reduces GKN1 and GKN2 expression in AGS gastric carcinoma cells, and that siRNA depletion of EBNA1 partially alleviates this repression. However, ectopic expression of EBNA1 slightly increased GKN1 and GKN2 basal mRNA levels, but reduced their responsiveness to demethylating agent.

Conclusions: These findings demonstrate that EBNA1 binds to the divergent promoter of the GKN1 and GKN2 genes in GC cells, and suggest that EBNA1 contributes to the complex transcriptional and epigenetic deregulation of the GKN1 and GKN2 tumor suppressor genes in EBV positive GC.
\end{abstract}

Keywords: EBV, EBNA1, Gastric carcinoma, Gastrokine, ChIP-Seq, Epigenetic

\section{Introduction}

Epstein-Barr virus (EBV) is a human gammaherpesvirus found in a wide range of lymphoid and epithelial cell malignancies, including Burkitt's lymphoma, Hodgkin's disease, nasopharyngeal carcinoma (NPC), and post-transplant lymphoproliferative disease (reviewed in [1,2]). More recently, EBV has been found in $\sim 10 \%$ of all gastric carcinoma (GC) cases worldwide [3,4]. EBV-associated GC has been shown to be a monoclonal outgrowth of EBV-infected gastric epithelial cells and is considered to be a distinct subtype of GC $[5,6]$. Because the incidence of $\mathrm{GC}$ is close to 900,000 people per year [7], EBV-associated GC may be among the most prevalent EBV-associated cancers.

In EBV positive gastric carcinoma cells, EBV establishes a variant type I latency, where EBV transcription is limited

\footnotetext{
* Correspondence: Lieberman@wistar.org

'The Wistar Institute, 3601 Spruce Street, Philadelphia, PA 19104, USA Full list of author information is available at the end of the article
}

to the canonical type I genes EBNA1, EBERs, BART family non-coding RNA and miRNAs, but with some additional expression of LMP2A [6,8-11]. Among these latency genes, EBNA1 is the only viral nuclear protein that is detected in EBV-associated GC. EBNA1 is required for the establishment of the latent episomal infection and for the long-term survival of latently infected cells [12-15]. EBNA1 is a DNA binding protein that binds to both viral and host chromosomal sites. The binding sites in the viral genome have been characterized for essential functions in replication and transcriptional control of viral gene expression. However, the function of EBNA1 sequence-specific binding to the host chromosome is less well understood. While EBNA1 can bind to the promoter regions of several host genes, it remains unclear whether these genes are subject to EBNA1 regulation [12,16,17]. Overexpression of the EBNA1 DNA binding domain, which functions as a dominant negative in EBV infected cells, can inhibit cell 
viability in uninfected cells, suggesting that EBNA1 binds to and regulates cellular genes important for cell survival [18]. Ectopic expression of EBNA1 has been shown to effect host cell mRNA expression [19], but it is not clear whether these effects are direct or indirectly related to specific EBNA1 binding sites in the cellular genome.

In a previous study, we used ChIP-seq methods to analyze the genome-wide enrichment sites of EBNA1 in latently infected Raji Burkitt's lymphoma cells and identified numerous cellular sites bound by EBNA1 [17]. Among those EBNA1 cellular enrichment sites we identified a significant EBNA1 binding peak located at the gastrokine 1 (GKN1) and gastrokine 2 (GKN2, also known as trefoil factor interacting protein (TFIZ1)) gene cluster. GKN1 and GKN2 have been identified based on their frequent loss of expression in neoplastic gastric carcinoma epithelial cells, compared to normal gastric tissue [20-22] (reviewed in [23]). Several recent studies have described anti-proliferative and anti-invasive activity for GKN1 in gastric epithelial cells, which, together with its frequent expression loss in cancer, suggests it functions as tumor suppressor specific to gastric epithelium [21,24-28]. GKN1 can inhibit cell migration and invasion in wound healing, transwell and Matrigel assay, as well as alter cell markers associated with the epithelial-mesenchymal transition [26]. GKN1 and GKN2 genes are located in close proximity and transcribed in opposite directions, suggesting that they likely share a bi-directional promoter, and are subject to coordinate regulation by shared transcription regulatory factors (reviewed in [23]).

In this study, we demonstrated the direct binding between EBNA1 and GKN1-GKN2 loci and investigated GKN1 and GKN2 gene expression modulation by EBV infection and EBNA1 protein. Our findings suggest that EBV infection can further inhibit GKN1 and GKN2 expression, and that loss of EBNA1 can facilitate epigenetic de-repression of GKN2 transcription. We also observed elevated DNA methylation levels at GKN1 and GKN2 promoter regions, and a potential role for EBNA1 in the deregulation and epigenetic repression of this tumor suppressor locus.

\section{Results}

\section{Identification of a high-occupancy EBNA1 binding site at} GKN1-GKN2 locus by ChIP-Seq

Previously published ChIP-Seq data from Raji BL cells revealed a limited number of highly enriched EBNA1 binding sites based on peak scores and read numbers [17]. Further inspection revealed a strong EBNA1 binding site located upstream of the start sites for the divergently transcribed GKN1 and GKN2 genes (Figure 1A, upper track). A similar EBNA1 binding peak was observed in a separate ChIP-Seq experiment performed in EBV positive nasopharyngeal carcinoma cell line C666-1 (full ChIP-seq data to be published elsewhere), indicating that this binding occurs in both epithelial, as well as lymphoid cell types (Figure 1A, lower track). The center of the peak was located $\sim 5 \mathrm{~kb}$ from GKN2 and $\sim 15 \mathrm{~kb}$ from the GKN1 transcription start sites. ChIP-qPCR was used to validate the EBNA1 binding at the GKN1-GKN2 promoter region in both Raji and C666-1 cells (Figure 1B and C). qPCR indicated that EBNA1 bound to the GKN12 site with similar efficiency to a previously validated EBNA1-binding site at the PITPNB promoter. qPCR also indicated that EBNA1 binding to GKN1-GKN2 was specific since there was no detectable EBNA1 binding at either the cellular GAPDH locus or EBV OriLyt control regions, as expected (Figure 1B and C). The putative EBNA1 binding site at GKN1-GKN2 locus was identified by alignment with a consensus binding site in the EBV family of repeats (FR) region, and this sequence was then tested for direct binding to EBNA1 by EMSA (Figure 1D). Purified recombinant EBNA1 DBD protein was assayed for binding to probes containing the GKN1-GKN2 site (GKN1/2), FR, or a negative control sequence lacking a consensus EBNA1 binding site. We found that EBNA1 DBD bound efficiently to the GKN1-GKN2 site, as well as to the FR consensus, but did not bind to the control sequence. These findings suggest that EBNA1 interacts with GKN1-GKN2 promoter region through direct DNA-binding with the EBNA1 DBD.

\section{GKN1 and GKN2 mRNA are highly expressed in primary stomach tissue}

We next measured GKN1 or GKN2 mRNA levels in various gastric carcinoma cells lines and in primary normal gastric tissue by qRT-PCR (Figure 2). We found that GKN1 and GKN2 are expressed at much higher levels $\left(\sim 3 \times 10^{4}\right.$ fold $)$ in primary stomach tissue than in any of the cell lines tested (Figure 2A). Although at much lower levels than primary stomach tissue, GKN1 and GKN2 were both expressed at measurable levels in AGS gastric carcinoma cell lines, with GKN2 expressed at higher levels than all other GC cell lines, or EBV positive B-cell or NPC cell lines (Figure 2B). Very low levels of GKN1 or GKN2 could be detected in primary oral epithelial tissue, further indicating that these genes are specific for primary gastrictissue.

\section{EBV latency reduces GKN1 and GKN2 mRNA expression in GC cell lines}

To test the effect of EBV latent infection on GKN1 and GKN2 expression, we generated an AGS cell line containing EBV B95.8 bacmid. The AGS cell line was selected for hygromycin resistance and GFP positivity to ensure that it contained bacmid components. To characterize the AGSEBV cell line, we first analyzed the EBV gene expression pattern (Figure 3). We found that AGS-EBV cells expressed detectable mRNA levels of EBNA1, BARF0, and LMP2A, 

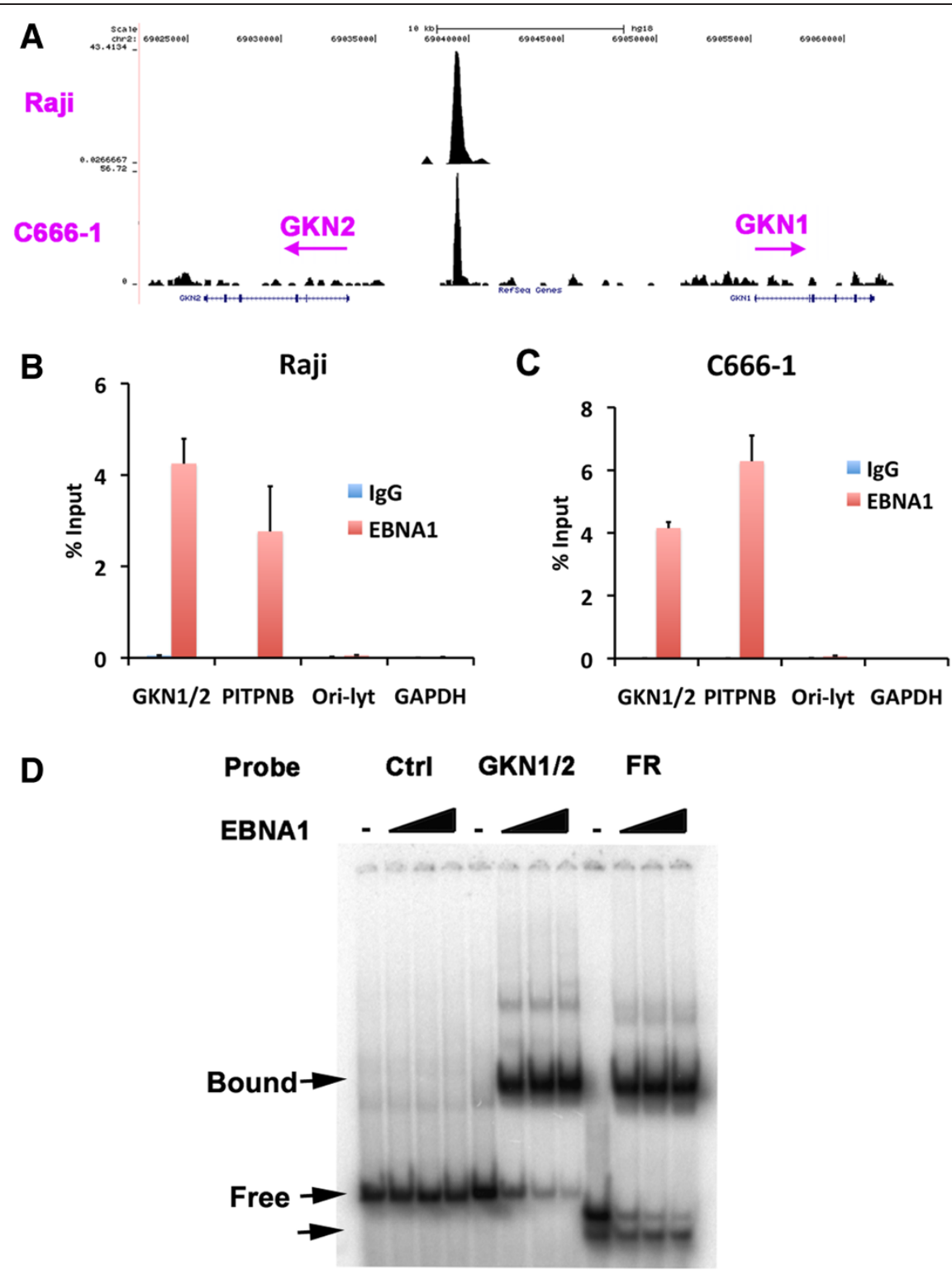

\section{GKN1/2 probe: TTTCTTATTTTCCAGGCAGCATATACTATCTCAAGCTCACCATGTC FR probe: GATCCGGATACAGATTAGGATAGCATATACTACCCA Ctrl probe: CGCCGCCGGGGCCTGCGGCGCCTCCCGCCCGGGCATGGGGCCGCGC}

Figure 1 EBNA1 binds at the GKN1 and GKN2 promoter locus. (A) The UCSC genome browser was used to map EBNA1 binding peak generated from Raji and C666-1 ChIP-seq at the GKN1 and GKN2 gene loci. RefSeq annotated transcripts are indicated below the ChIP-Seq peak. (B-C) Realtime-PCR validation of ChIP-seq data for EBNA1 binding site at the GKN1/2 shared promoter region: EBNA1 (red bars) or control lgG (blue bars) were assayed by ChIP in Raji (B) or C666-1 cells (C) for DNA binding at the GKN1/2 site, PITPNB promoter, GAPDH, or EBV Ori-Lyt. (D) EMSA analysis of ${ }^{32} \mathrm{P}$ labeled probes containing Control, GKN1/2 site, or EBV FR. Varying amount of EBNA1 DBD protein was used in binding reaction $(0,100,300,900 \mathrm{ng})$. Arrowheads represent EBNA1-specific bound complexes or free probe as indicated. The probe sequence of GKN1/2 site and FR is indicated with sequence homolog (red letters) between GKN1/2 and FR. The negative control (Ctrl) sequence is also indicated. Error bars indicate standard deviation from the mean $(\mathrm{sdm})$ for $\mathrm{n}=3$.

but not LMP1, EBNA2, or EBNA3C (Figure 3). Although EBV B95.8 bacmid lacks the BART miRNAs, these findings are consistent with AGS-EBV cells adopting a variant type I latency gene expression program with some expression of
LMP2A, similar to that observed in EBV positive gastric carcinoma tumor tissue $[8,10]$. To further characterize AGS-EBV cells, we analyzed EBNA1 protein expression by Western blot (Figure 4A) and EBV DNA copy number by 


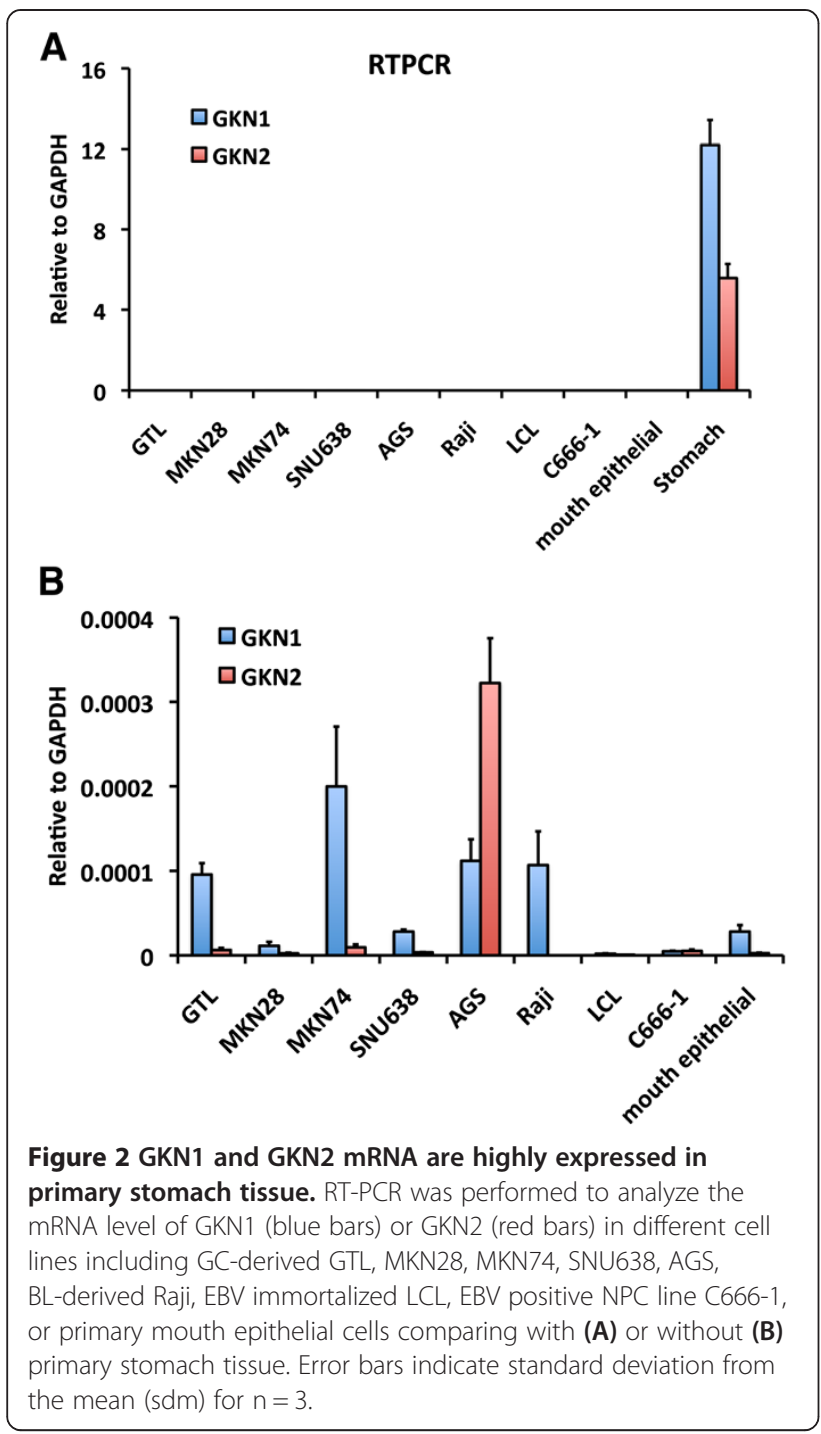

qPCR (Figure 4B). EBNA1 was detected as a single low abundance species at the expected molecular mass (Figure 4A). EBV copy number was measured by comparing EBV Ori-Lyt DNA to cellular GAPDH (Figure 4B). We found that AGS-EBV contained $\sim 50 \%$ less copies of the viral genome compared to EBV positive LCLs. To determine if EBNA1 retained its DNA binding activity in AGSEBV, we performed conventional ChIP assays (Figure 4C). We found that EBNA1 bound to the EBV Dyad Symmetry (DS) DNA, as well as to the GKN1-GKN2 binding site in AGS-EBV cells. We next asked whether GKN1 or GKN2 mRNA levels were affected by EBV latent infection in AGS cells by comparing RT-qPCR expression levels in AGS relative to AGS-EBV cells (Figure 4D). We found that GKN1 and GKN2 were repressed $\sim 3-8$ fold in AGS-EBV relative to AGS cells, suggesting that EBV latency promotes or stabilizes the transcriptional repression of GKN1 and GKN2.
EBV increases DNA methylation dependent repression of GKN1 and GKN2 mRNA in GC cell lines

GKN1 and GKN2 may be subject to epigenetic suppression in GC and tissue culture cell lines. To explore this possibility, we tested whether treatment with DNA demethylating agent $5^{\prime}$ azacytidine (Aza) or deacetylating agent $(\mathrm{NaB})$ combined with phorbol ester (TPA) would activate GKN1 or GKN2 in AGS cells (Figure 5A). We found that Aza treatment led to a $\sim 10$ fold increase in GKN2, and $\sim 4$ fold increase in GKN1 transcription in AGS treated cells. In contrast, $\mathrm{NaB} / \mathrm{TPA}$ treatment produced only $\sim 2$ fold activation of transcription. These findings suggest that both GKN1 and GKN1 are under active epigenetic repression through DNA methylation. To test if EBV had any effect on Aza induced activation of GKN1 or GKN2, we compared the effects of Aza treatment on AGS relative to AGS-EBV cells by qRT-PCR (Figure 5B). We found that GKN1 and GKN2 were efficiently activated by Aza treatment in AGS cells, but to a lesser extent in AGS-EBV. In these experiments, Aza-induced activation of GKN2 was completely eliminated, while activation of GKN1 was only partly attenuated. Aza-treatment also led to the $\sim 8.4$ fold increase in EBNA1 mRNA levels, suggesting that Aza treatment stimulates EBV lytic gene expression in AGS-EBV cell lines. These results suggest the EBV gene products prevent GKN2, and to a lesser extent GKN1 activation after Aza treatment.

To better understand the mechanism of GKN1 and GKN2 epigenetic repression, we examined the DNA methylation levels using methyl cytosine-specific antibody directed DNA immunoprecipitation (MeDIP) assay. We assayed the enrichment of methylated DNA in the GKN1GKN2 control region in AGS and AGS-EBV cells with or without Aza treatment (Figure 5C). We observed a relative enrichment of methylated $\mathrm{CpG}$ at a region between the EBNA1 binding site and GKN2 transcription start site (GKN2_A). Aza treatment led to a decrease in MeDIP signal in most regions where a signal was detected, suggesting that Aza treatment led to a general reduction in DNA methylation. Similar loss of CpG methylation was observed at the alpha-globin gene (which does not bind EBNA1), and at several EBNA1 binding sites, including HDAC3 and MAP3K7IP (Figure 5D). We also noted that MeDIP signals were generally higher in EBV-AGS than in AGS cells, suggesting that EBV latent infection may promote or stabilize DNA methylation throughout the host genome.

\section{EBNA1 depletion activates GNK1 and GKN2 mRNA expression in EBV positive epithelial cells}

To determine if EBNA1 contributed to the transcriptional repression of GKN1 and GKN2, we first attempted to deplete EBNA1 in AGS-EBV cells using siRNA (Figure 6). We generated a siRNA targeting the 3 ' non-coding UTR 

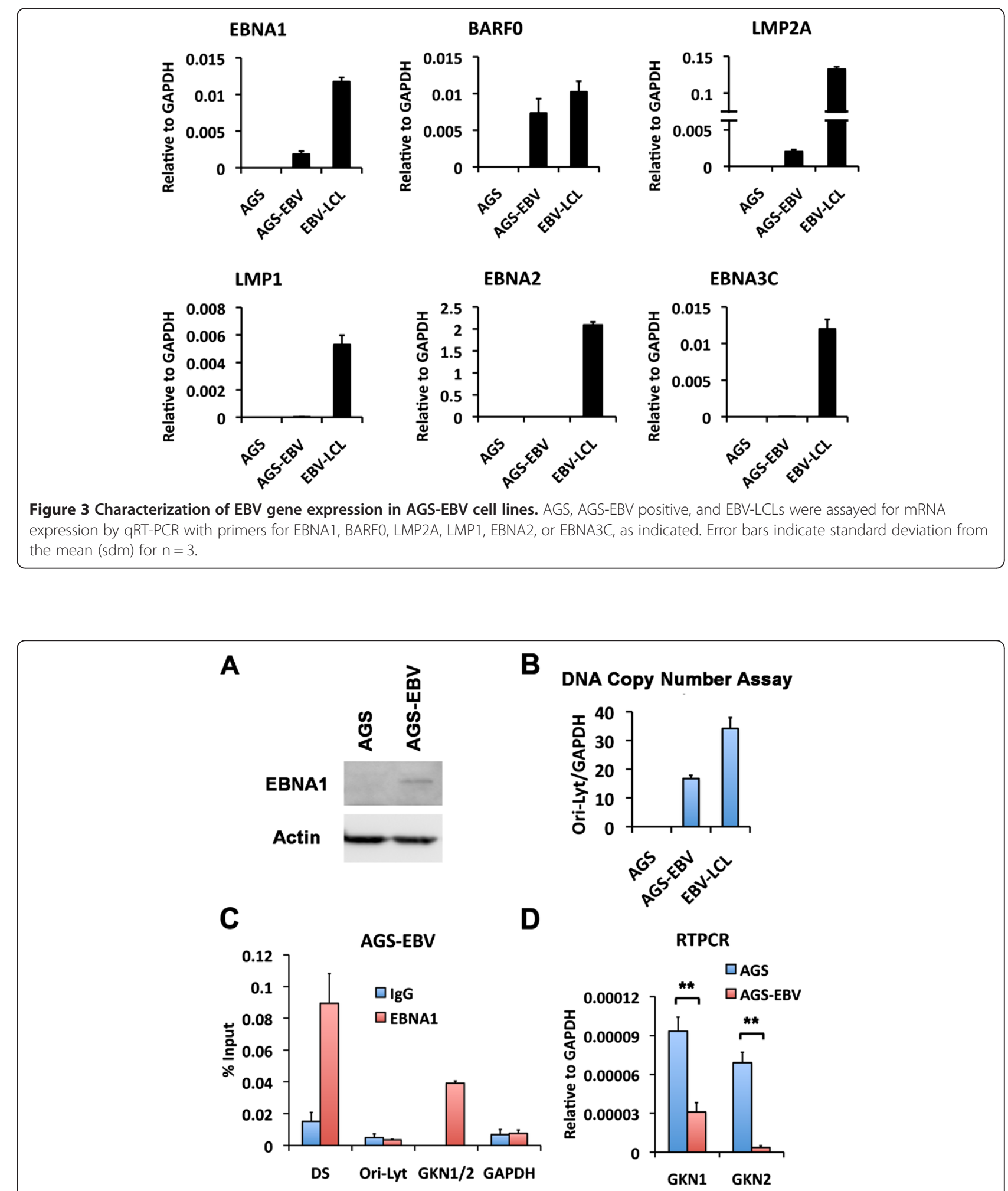

Figure 4 EBV infection of AGS cells suppresses GKN1 and GKN2 transcription. (A) Western blot analysis of EBNA1 protein expression in AGS-EBV cells compared with EBV-negative AGS cells was performed using antibody for EBNA1 (top) or Actin (bottom). (B) DNA copy number was assayed by real-time PCR of EBV Ori-Lyt DNA relative to the level of cellular GAPDH in AGS, AGS-EBV, and EBV-LCL cells. (C) ChIP and real-time PCR analysis of EBNA1 (red bars) or control IgG (blue bars) for DNA binding at the EBV sites including DS and Ori-Lyt, or cellular sites including GKN1/2 and GAPDH in AGS-EBV cells. (D) RT-PCR was performed to analyze the mRNA level of GKN1 or GKN2 in AGS (blue bars) or AGS-EBV cells (red bars). ${ }^{* *}$ indicates $p<.005$. Error bars indicate standard deviation from the mean $(\mathrm{sdm})$ for $n=3$. 


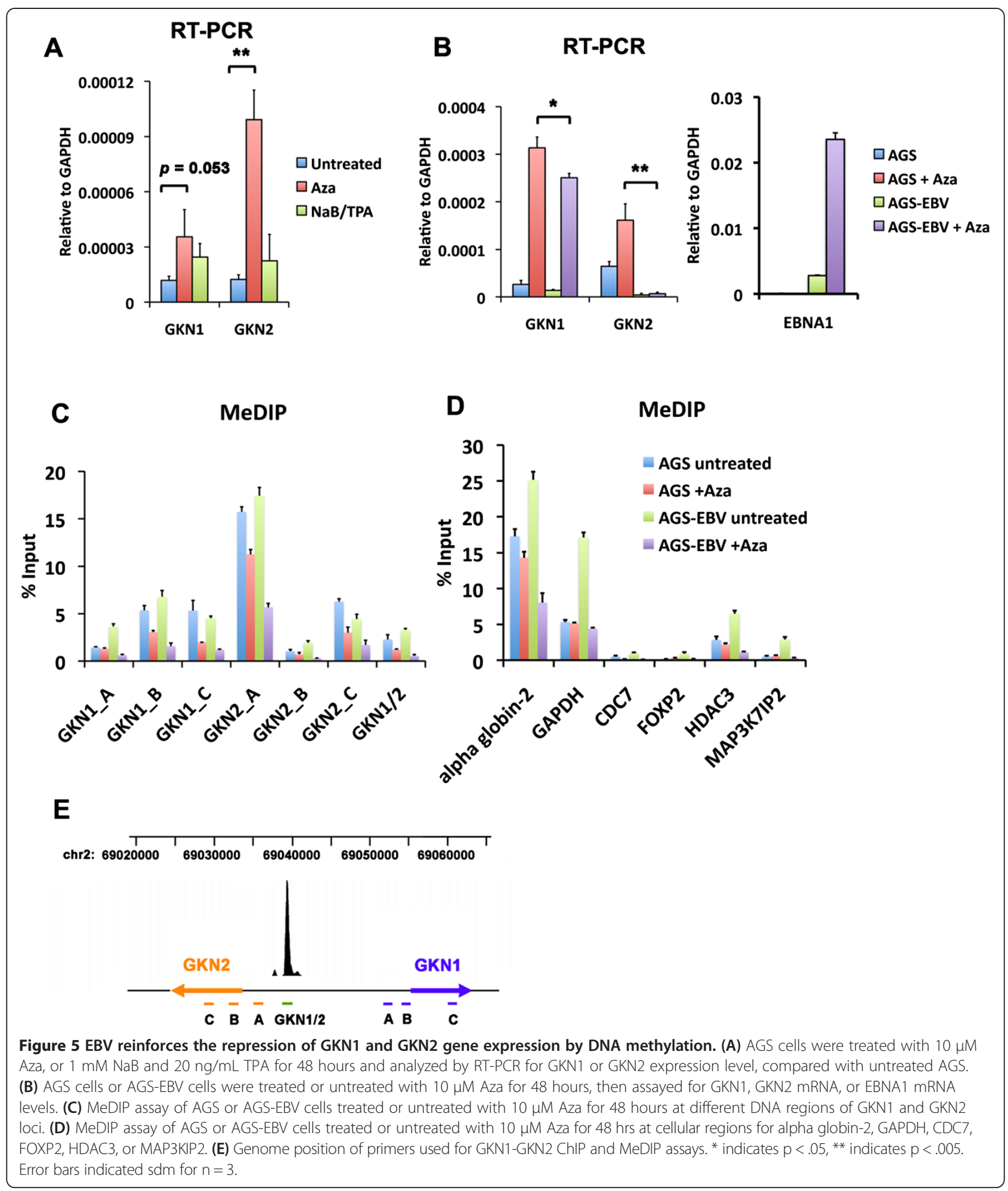

of EBNA1 mRNA, which partially depleted EBNA1 protein in AGS-EBV cells (Figure 6B). Depletion of EBNA1 resulted in an $\sim 5$ fold activation of GKN2, with little detectable activation of GKN1 (Figure 6A). These findings suggest that EBNA1 may function as a transcriptional repressor of GKN2 in AGS-EBV cells. 


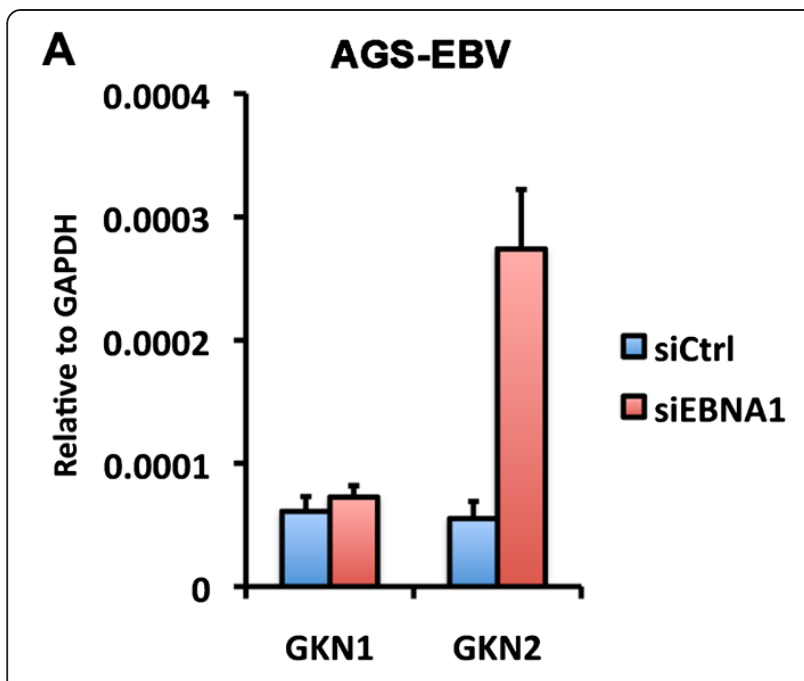

B

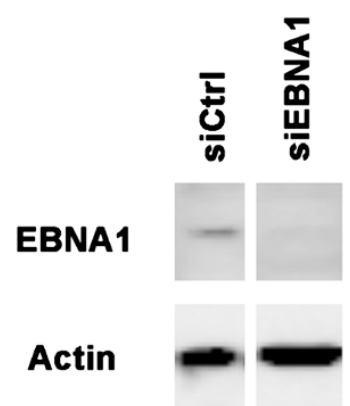

Figure 6 siRNA depletion of EBNA1 causes de-repression of the GKN1 and GKN2 in AGS-EBV cells. siCtrl or siEBNA1 transfected AGS-EBV cells were assayed by RT-PCR for GKN1 or GKN2 mRNA level relative to cellular GAPDH (A). Cells were harvested at 72 hours post-transfection with siRNA. Western blot showing EBNA1 (top panel) and loading control Actin (lower panel) in AGS-EBV cells (B).

Error bars indicate standard deviation from the mean $(\mathrm{sdm})$ for $n=3$.

EBNA1 inhibits GKN1 and GKN2 transcription after DNA demethylation

To further explore the contribution of EBNA1 to GKN1 and GKN2 transcription regulation, we tested the effect of ectopic expression of EBNA1 alone on Aza-induced levels of GKN1 or GKN2 transcription in GC cells. AGS cells were transduced with an EBNA1 expressing lentivirus. Stable AGS-EBNA1 (pLU-EBNA1) or AGS- control vector (pLU-Vec) cell lines were selected and assayed for GKN1 and GKN2 mRNA levels. We observed that AGS-EBNA1 cells had a $\sim 2$-3 fold higher basal level of GKN1 and GKN2 mRNA relative to parental AGS cells (Figure 7A). However, Aza-induced mRNA levels of GKN1 and GKN2 were attenuated in AGSEBNA1 compared to AGS cells (Figure 7B). Aza treatment also led to a large increase in EBNA1 mRNA levels (Figure 7C). To determine if the effects of EBNA1 on GKN1 and GKN2 were specific to AGS cells, we transduced another EBV negative GC cell line MKN74 with
EBNA1 lentivirus (Figure 7D-F). Similar to AGS cells, we found that EBNA1 increased basal levels of GKN1 and GKN2, but inhibited the ability of Aza to further induce GKN1 and GKN2 mRNA levels (Figure 7D and E). We confirmed that Aza-treatment was effective by measuring EBNA1 mRNA levels, which increased $\sim 7$ fold (Figure 7F). These findings suggest that ectopic expression of EBNA1 can increase basal, but inhibit Azainduced levels of GKN1 and GKN2 transcription in EBV-negative GC cell lines.

\section{Discussion}

In this study, we identified a high-occupancy EBNA1 binding site in the $5^{\prime}$ promoter control region of the divergently transcribed GKN1 and GKN2 genes. EBNA1 binding sites were observed in two independent ChIP-Seq data sets from EBV positive lymphoid BL cells Raji and EBV positive epithelial nasopharyngeal carcinoma cells C666-1 (Figure 1A). We confirmed these binding sites by conventional ChIP-qPCR in both cell lines (Figure $1 \mathrm{~B}$ and $\mathrm{C}$ ). EBNA1 was also shown to bind directly to these sites by EMSA with purified recombinant EBNA1 DBD protein (Figure 1D). We show that GKN1 and GKN2 mRNA levels are highly repressed in most cell lines relative to primary gastric tissue (Figure 2). To study the potential role of EBV and EBNA1 in the transcriptional control of GKN1 and GKN2, we generated an EBV positive AGS gastric carcinoma cell line. We show that EBV adopts a variant type I latency pattern in AGS cells (Figure 3), and that EBNA1 can bind to the GKN1/GKN2 promoter region in the cellular chromosome (Figure 4C). We also found that GKN1 and GKN2 mRNA were further suppressed in EBV positive AGS cells relative to control EBV negative AGS cells (Figure 4D). We then showed that Aza-treatment led to the increase expression of GKN1 and GKN2 (Figure 5A), and that EBV latent infection inhibits Aza activation of GKN2 (Figure 5B). We found that siRNA depletion of EBNA1 in EBV positive AGS cells leads to transcription activation of GKN2 (Figure 6). We also show that EBNA1 ectopic expression moderately increases basal, but inhibits the Aza-induced levels of GKN1 and GKN2 transcription (Figure 7). Taken together, these findings indicate that EBNA1 binds to the GKN1-GKN2 promoter control region in multiple cell types, and raise the possibility that EBNA1 contributes to the transcriptional and epigenetic repression of the GKN1 and GKN2 tumor suppressor genes in EBV positive GC.

EBV latent infection is known to increase the tumorigenic phenotype of gastric carcinoma cells [29-31]. GKN1 and GKN2 are reported to function as cell growth inhibitors and tumor suppressors in GC [20,21,23,25-27]. Our mRNA expression data showing high-level mRNA expression only in primary normal gastric tissue are consistent with a role of GKN1 and GKN2 as a tumor suppressor. 


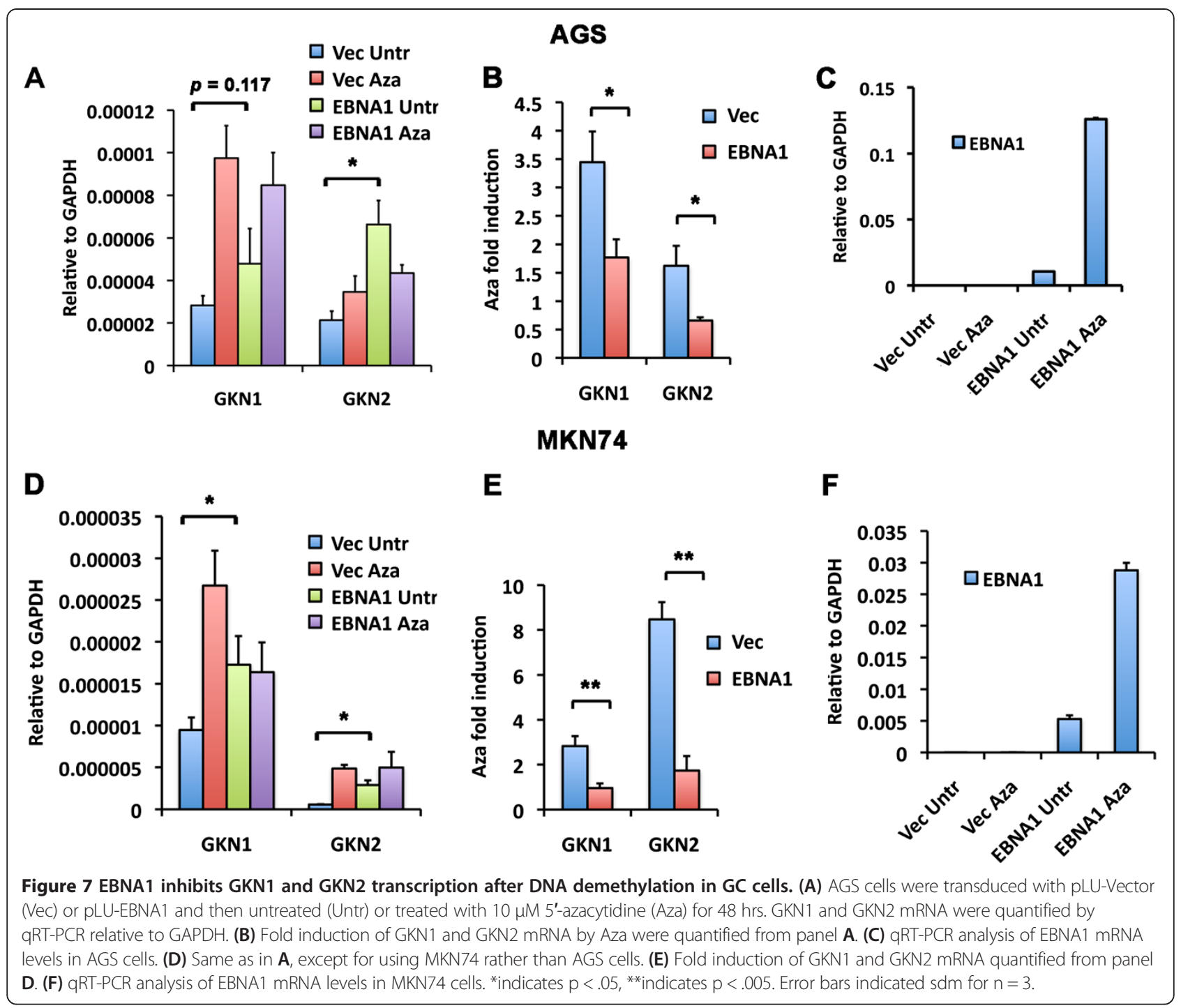

However, we were unable to show that over-expression of either or both GKN1 or GKN2 in AGS or AGS-EBV cause a cell cycle arrest or reduce viability (data not shown). This suggests that GKN1 and GKN2 function at earlier stages in tumor cell evolution, or in more complex tumor microenvironments. We speculate that EBNA1 may have a more pronounced effect on GKN1 and GKN2 expression in situations where EBV may infect primary gastric cells where basal expression of GKN1 and GKN2 are high and important for tumor suppression.

Previous published studies have shown that GKN1 and GKN2 transcription is subject to epigenetic suppression by DNA methylation in all forms of GC [21]. Our studies are consistent with the role of DNA methylation in the epigenetic suppression of GKN1 and GKN2 in AGS cells. Treatment with Aza resulted in the 4-10 fold increase in GKN1 and GKN2 mRNA expression (Figure 5A), and MeDIP revealed enrichment of methylated DNA at the promoter regions (Figure $5 \mathrm{C}$ ). AGS-EBV cells did show an increase in DNA methylation at several cellular sites, including regions surrounding the EBNA1 binding sites at the GKN1 promoter region (Figure 5C), and the HDAC3 and MAP3K7IP2 genes (Figure 5D). However, the presence of EBNA1 in AGS-EBV cells did not prevent Aza-induced demethylation at these sites. This suggests that EBNA1 may repress transcription from some promoters, like GKN2, through a mechanism distinct from DNA methylation. However, ectopic expression of EBNA1 alone produced a more complicated phenotype, causing a small increase in basal expression, but limiting the effects of Aza-induced demethylation (Figure 7). This may suggest that that EBNA1 may function differently when expressed ectopically, than when expressed in the context of the viral genome. Nevertheless, our findings suggest that EBNA1 perturbs the normal transcriptional regulation of the GKN1 and GKN2 genes. 
The precise function of EBNA1 in transcription regulation remains unclear. EBNA1 has been implicated in the transcriptional activation and repression of both viral and cellular genes [32,33]. EBNA1 can repress its own mRNA expression from the EBV Qp in type III latency, where repression has been linked to steric interference with RNA polymerase II binding to the transcription initiation site [34]. On the other hand, EBNA1 can activate $\mathrm{Cp}$ and LMP1 promoters in type III latency where it may function as an enhancer-like factor [35-37]. EBNA1 has been implicated in transcription activation of some cellular genes, including the Nox2 gene involved in reactive oxygen species formation [19]. EBNA1 may also affect host-cell transcription through a global remodeling of the host chromosome [38]. Thus, EBNA1 may alter cellular transcription through multiple direct and indirect mechanisms.

Epigenetic modifications are known to play an important role in EBV-associated gastric carcinoma [39]. Interestingly, AGS cells carrying EBV bacmid genomes had higher levels of methylated DNA at many tested sites (Figure 5D). This is consistent with the proposed role of EBV in the methylation of host tumor suppressor genes [40]. This is also consistent with the findings that EBV positive GC has elevated DNA methylation at promoter regions of several key GC tumor suppressors, including gastrokine genes [39,41-45]. While EBNA1 bound near DNA methylated regions of the GKN2, we were unable to show that EBNA1 modulates DNA methylation at the GKN1 and GKN2 sites (data not shown). However, it is possible that EBNA1 in association with another viral encoded or induced factor may stabilize GKN1 and GKN2 transcriptional repression through a chromatin-dependent and structural mechanism that reinforces DNA methylation. It is also possible that EBNA1 may regulate GKN1 or GNK2 only in tissue or tumor microenvironments that are not readily recapitulated in cell culture. While the function of EBNA1 binding to host cell chromosome sites remains an important area of investigation, more sophisticated infection models may be required to elucidate its potential role in altering host cell gene expression and carcinogenesis.

\section{Methods}

\section{Cells, plasmids, and lentivirus infection}

EBV-positive Burkitt's lymphoma Raji cells, EBV positive nasopharyngeal carcinoma C666-1 cells, and gastric carcinoma cell lines (a gift from Dr. Antonia R. Sepulveda, Columbia University) including GTL, MKN28, MKN74, SNU638 were maintained in RPMI containing 10\% FBS and supplemented with antibiotics (penicillin and streptomycin). Gastric carcinoma AGS cells (ATCC No. CRL-1739) were maintained in F-12K containing $10 \%$ FBS. Primary mouth epithelial cells were provided by Dr. Manjunatha Benakanakere, University of Pennsylvania and cultured in Keratinocyte-SFM medium. EBV-LCL was established by primary infection of peripheral blood mononuclear cells (PBMC) with EBV BAC virions generated from stimulated 293-EBV cells [46,47]. EBV-LCL contains a hygromycin B resistant EBV bacmid were maintained in RPMI containing 10\% FBS, hygromycin B $(100 \mu \mathrm{g} / \mathrm{ml})$, glutamax (Invitrogen), and antibiotics. AGSEBV cells were generated from AGS cells co-cultivated with EBV-LCL by adapting a previously published cocultivation method described AGS cells infection with rEBV through cell-to-cell contact [48] with some modification. Briefly, EBV-LCL was induced by $20 \mathrm{ng} / \mathrm{mL} 12-\mathrm{O}-$ tetradecanoylphorbol-13-acetate (TPA) and $1 \mathrm{mM}$ sodium butyrate $(\mathrm{NaB}) 24$ hours prior to co-cultivation. The induced EBV-LCL cells were washed with PBS twice to completely remove the inducing agents, resuspended with complete RPMI medium at $10^{6}$ cells $/ \mathrm{ml}$ before the coincubation. AGS cells were plated in 6-well Plates 24 hours before co-cultivation, then 60 - 70\% confluent AGS cells in $1 \mathrm{ml}$ complete F-12K medium were incubated with $10^{6}$ induced and washed EBV-LCL cells in $1 \mathrm{ml}$ complete RPMI. 24 hours later, $2 \mathrm{ml}$ of Serum free F-12K medium was added to each well to reduce the FBS concentration to $5 \%$ to prevent cell overgrowth. 3 days after coincubation, EBV-LCL cells were removed from the cocultures and the AGS cells were thoroughly washed with PBS at least 5 times to remove any of the donor EBV-LCL cells. The infected AGS cells was then incubated with fresh F-12K medium with 10\% FBS and $100 \mu \mathrm{g} / \mathrm{ml}$ hygromycin $B$ and the selection medium was changed every 2 to 3 days until the infected AGS cells formed Hyg B selection colonies with GFP expression (usually 3 to 4 weeks after co-cultivation). The selection colonies were then pooled and tested for EBNA1 expression and EBV genome copy number before subject to experiments. AGS-EBV cells were maintained in F-12K medium with $10 \%$ FBS and $100 \mu \mathrm{g} / \mathrm{ml}$ hygromycin B.

pLU-EBNA1 Lentivirus expression vector was constructed by PCR amplification of EBNA1 with primers (GCGGGATCCTCTGACGAGGGGCCAGGTACAG GACCT and ATCGTCGACTCACTCCTGCCCTTCCT CACCCTCATC) introducing a 5' BamH I and 3' Sal I site cloned in frame to pLU-TCMV-FMCS-pPURO. AGS or MKN74 cells were infected with Lentivirus expressing pLU-EBNA1 or pLU control vector generated freshly from 293T cells. Infected AGS or MKN74 cells were then selected with $2.5 \mu \mathrm{g} / \mathrm{ml}$ Puromycin for 10 to 14 days. The selected cells were pooled and treated with or without 5 'azacytidine for 48 hours then subjected to RT-PCR.

siRNA against EBNA1 and siRNA Control (Cat. No. D001810-01-20) were all purchased from Dharmacon. siRNA directed against EBNA1 3'UTR were synthesized using the target sequence CGGAGAUGACGGAGAUG AAUU. Transfection of siRNA duplexes was conducted by 
using Oligofectamine (Dharmacon), following manufacturers specifications.

\section{Chromatin immunoprecipitation (ChIP) assays}

ChIP assays were performed as described previously [49]. Quantification of precipitated DNA was determined using real-time PCR and the delta Ct method for relative quantification (ABI 7900HT Fast Real-Time PCR System; Applied Biosystems). Primers for ChIP assays are available upon request. The following antibodies were used for ChIP assays: rabbit anti-EBNA1 (305/10 wk), anti- rabbit IgG (Santa Cruz Biotechnology, sc-2027). Anti-Actin HRP (Sigma, A3854) and rabbit anti-EBNA1 (305/10 wk) were used for Western Blotting. EBNA1 specific antibody was raised against a bacterial EBNA1 protein lacking the GArepeat region using New Zealand white rabbits and then affinity purified using the same bacterial protein coupled to sepharose beads.

\section{EMSA}

DNA fragments were labeled using T4 polynucleotide kinase (NEB) in the presence of $\left[\gamma^{3}{ }^{32} \mathrm{p}\right]$ ATP and purified using G-25 spin columns (GE Healthcare). Purified EBNA1 DNA binding domain [17] was incubated with probes at room temperature for $30 \mathrm{~min}$ in a total volume of $20 \mu \mathrm{l}$ buffer containing 10mM TrisCl PH 8.0, $100 \mathrm{mM}$ $\mathrm{KCl}, 1 \mathrm{mM}$ EDTA, $10 \mathrm{mM} \mathrm{MgCl}, 0.05 \mu \mathrm{g} / \mu \mathrm{l}$ poly $(\mathrm{dI}-\mathrm{dC})$, $0.5 \mu \mathrm{g} / \mu \mathrm{l}$ bovine serum albumin, $0.05 \% \mathrm{NP} 40,35 \mathrm{mM} \beta$ mercaptoethanol, and $10 \%$ glycerol. The samples were then separated by electrophoresis on a native $5 \%$ polyacrymide gel. Gels were dried and analyzed using a Typhoon phosphorImager system. The forward probe sequences are gatccggatacagattaggatagcatatactaccca (FR), tttcttattttccagg cagcatatactatctcaagctcaccatgtc (GKN1/2) and cgccgccgggg cctgcggcgcctcccgcccgggcatggggccgcgc (negative control, LANA binding sites at KSHV TR).

\section{Quantitative RT-PCR}

For cell lines, RNA was isolated from $2 \times 10^{6}$ cells using RNeasy Kit (Qiagen) and then further treated with DNase I. Reverse transcriptase PCR (RT-PCR) was performed as previously described [50]. Real-time PCR was performed with SYBR green probe in an ABI Prism 7900 according to the manufacturer's specified parameters. Primer sequences for RT-PCR are available upon request. Total human stomach RNA (Cat. No. 540037) was purchased from Agilent Technologies.

\section{Genome copy number assay}

Genome copy number assay was performed as described previously [51]. The total cellular DNA was assayed by real-time PCR using primers for the EBV Ori-Lyt region and normalized by the cellular GAPDH
DNA region. The primers for Ori-Lyt and GAPDH are available upon request.

\section{MeDIP}

Methyl-DNA immunoprecipitation (MeDIP) methods have been described previously [51]. MeDIP DNA were analyzed by real-time PCR with delta Ct method for quantification. The primers for MeDIP are available upon request.

\section{Competing interests \\ The authors declare no competing interests, with the exception that PML declares an interest in Vironika, LLC that is developing small molecule inhibitors for EBNA1.}

\section{Author contributions}

FL and PML conceived of the study, developed its design, and drafted the manuscript. FL carried out the molecular genetic studies. IT participated in ChIP-seq studies. HTL participated in cell-based assays. KD carried out RTPCR. All authors read and approved the final manuscript.

\section{Acknowledgments}

We thank Andreas Wiedmer for technical assistance. We thank H.J. Delecluse for kindly providing EBV bacmid. We acknowledge the support of the Wistar Institute Cancer Center Core Facilities in Flow Cytometry, Bioinformatic, and Genomics. This work was supported by Wistar Institute Cancer Center Core Grant (P30 CA10815), K99AI099153 award from the National Institute Of Allergy And Infectious Diseases to IT, and RO1 (CA085678, CA093606, and DE017336) to PML.

\section{Author details}

${ }^{1}$ The Wistar Institute, 3601 Spruce Street, Philadelphia, PA 19104, USA. 2Department of Microbiology, The Fels Cancer Institute, Temple University School of Medicine, Philadelphia, PA, USA.

Received: 28 October 2013 Accepted: 17 January 2014

Published: 24 January 2014

\section{References}

1. Kieff E: Epstein-Barr virus and its replication. 5th edition. Philadelphia: Wolters Kluwer Health/Lippincott Williams \& Wilkins; 2007.

2. Rickinson AB, Kieff E: Epstein-Barr virus. 5th edition. Philadelphia: Wolters Kluwer Health/Lippincott Williams \& Wilkins; 2007.

3. Takada K: Epstein-Barr virus and gastric carcinoma. Mol Pathol 2000, 53:255-261.

4. Fukayama M, Ushiku T: Epstein-Barr virus-associated gastric carcinoma. Pathol Res Pract 2011, 207:529-537.

5. Fukayama M: Epstein-Barr virus and gastric carcinoma. Pathol Int 2010, 60:337-350.

6. Imai S, Koizumi S, Sugiura M, Tokunaga M, Uemura Y, Yamamoto N, Tanaka S, Sato E, Osato T: Gastric carcinoma: monoclonal epithelial malignant cells expressing Epstein-Barr virus latent infection protein. Proc Natl Acad Sci USA 1994, 91:9131-9135.

7. Crew KD, Neugut Al: Epidemiology of upper gastrointestinal malignancies. Semin Oncol 2004, 31:450-464.

8. Zur Hausen A, Brink AA, Craanen ME, Middeldorp JM, Meijer CJ, van den Brule AJ: Unique transcription pattern of Epstein-Barr virus (EBV) in EBVcarrying gastric adenocarcinomas: expression of the transforming BARF1 gene. Cancer Res 2000, 60:2745-2748.

9. Sugiura M, Imai S, Tokunaga M, Koizumi S, Uchizawa M, Okamoto K, Osato $\mathrm{T}$ : Transcriptional analysis of Epstein-Barr virus gene expression in EBVpositive gastric carcinoma: unique viral latency in the tumour cells. $\mathrm{Br} J$ Cancer 1996, 74:625-631

10. Luo B, Wang Y, Wang XF, Liang H, Yan LP, Huang BH, Zhao P: Expression of Epstein-Barr virus genes in EBV-associated gastric carcinomas. World J Gastroenterol 2005, 11:629-633.

11. Oh ST, Seo JS, Moon UY, Kang KH, Shin DJ, Yoon SK, Kim WH, Park JG, Lee SK: A naturally derived gastric cancer cell line shows latency I Epstein- 
Barr virus infection closely resembling EBV-associated gastric cancer. Virology 2004, 320:330-336.

12. Smith DW, Sugden B: Potential cellular functions of Epstein-Barr Nuclear Antigen 1 (EBNA1) of Epstein-Barr virus. Viruses 2013, 5:226-240.

13. Lindner SE, Sugden B: The plasmid replicon of Epstein-Barr virus: mechanistic insights into efficient, licensed, extrachromosomal replication in human cells. Plasmid 2007, 58:1-12.

14. Frappier L: Contributions of Epstein-Barr nuclear antigen 1 (EBNA1) to cell immortalization and survival. Viruses 2012, 4:1537-1547.

15. Frappier L: Role of EBNA1 in NPC tumourigenesis. Semin Cancer Biol 2012 , 22:154-161

16. Dresang $L R$, Vereide DT, Sugden B: Identifying sites bound by Epstein-Barr virus nuclear antigen 1 (EBNA1) in the human genome: defining a position-weighted matrix to predict sites bound by EBNA1 in viral genomes. J Virol 2009, 83:2930-2940.

17. Lu F, Wikramasinghe P, Norseen J, Tsai K, Wang P, Showe L, Davuluri RV Lieberman PM: Genome-wide analysis of host-chromosome binding sites for Epstein-Barr Virus Nuclear Antigen 1 (EBNA1). Virol J 2010, 7:262.

18. Mack $A A$, Sugden $B$ : EBV is necessary for proliferation of dually infected primary effusion lymphoma cells. Cancer Res 2008, 68:6963-6968.

19. Gruhne B, Sompallae R, Marescotti D, Kamranvar SA, Gastaldello S, Masucci MG: The Epstein-Barr virus nuclear antigen-1 promotes genomic instability via induction of reactive oxygen species. Proc Natl Acad Sci USA 2009, 106:2313-2318.

20. Moss SF, Lee JW, Sabo E, Rubin AK, Rommel J, Westley BR, May FE, Gao J, Meitner PA, Tavares R, Resnick MB: Decreased expression of gastrokine 1 and the trefoil factor interacting protein TFIZ1/GKN2 in gastric cancer: influence of tumor histology and relationship to prognosis. Clin Cancer Res 2008, 14:4161-4167.

21. Yoon JH, Song JH, Zhang C, Jin M, Kang YH, Nam SW, Lee JY, Park WS: Inactivation of the Gastrokine 1 gene in gastric adenomas and carcinomas. J Pathol 2011, 223:618-625.

22. Oien KA, McGregor F, Butler S, Ferrier RK, Downie I, Bryce S, Burns S, Keith WN: Gastrokine 1 is abundantly and specifically expressed in superficial gastric epithelium, down-regulated in gastric carcinoma, and shows high evolutionary conservation. J Pathol 2004, 203:789-797.

23. Menheniott TR, Kurklu B, Giraud AS: Gastrokines: stomach-specific proteins with putative homeostatic and tumor suppressor roles. Am J Physiol Gastrointest Liver Physiol 2013, 304:G109-G121.

24. Mao W, Chen J, Peng TL, Yin XF, Chen LZ, Chen MH: Role of trefoil factor 1 in gastric cancer and relationship between trefoil factor 1 and gastrokine 1. Oncol Rep 2012, 28:1257-1262.

25. Mao W, Chen J, Peng TL, Yin XF, Chen LZ, Chen MH: Downregulation of gastrokine-1 in gastric cancer tissues and restoration of its expression induced gastric cancer cells to apoptosis. J Exp Clin Cancer Res 2012, 31:49.

26. Yoon JH, Kang YH, Choi YJ, Park IS, Nam SW, Lee JY, Lee YS, Park WS: Gastrokine 1 functions as a tumor suppressor by inhibition of epithelial-mesenchymal transition in gastric cancers. J Cancer Res Clin Oncol 2011, 137:1697-1704.

27. Xing R, Li W, Cui J, Zhang J, Kang B, Wang Y, Wang Z, Liu S, Lu Y: Gastrokine 1 induces senescence through p16/Rb pathway activation in gastric cancer cells. Gut 2012, 61:43-52.

28. Yan GR, Xu SH, Tan ZL, Yin XF, He QY: Proteomics characterization of gastrokine 1-induced growth inhibition of gastric cancer cells. Proteomics 2011, 11:3657-3664.

29. Marquitz AR, Mathur A, Shair KH, Raab-Traub N: Infection of Epstein-Barr virus in a gastric carcinoma cell line induces anchorage independence and global changes in gene expression. Proc Natl Acad Sci USA 2012, 109:9593-9598.

30. Cheng TC, Hsieh SS, Hsu WL, Chen YF, Ho HH, Sheu LF: Expression of Epstein-Barr nuclear antigen 1 in gastric carcinoma cells is associated with enhanced tumorigenicity and reduced cisplatin sensitivity. Int $J$ Oncol 2010, 36:151-160.

31. Hino R, Uozaki H, Inoue Y, Shintani Y, Ushiku T, Sakatani T, Takada K, Fukayama M: Survival advantage of EBV-associated gastric carcinoma: survivin up-regulation by viral latent membrane protein $2 \mathrm{~A}$. Cancer Res 2008, 68:1427-1435.

32. Canaan A, Haviv I, Urban AE, Schulz VP, Hartman S, Zhang Z, Palejev D, Deisseroth AB, Lacy J, Snyder M, et al: EBNA1 regulates cellular gene expression by binding cellular promoters. Proc Natl Acad Sci USA 2009, 106:22421-22426
33. Kennedy G, Sugden B: EBNA-1, a Bifunctional Transcriptional Activator. Mol Cell Biol 2003, 23:6901-6908.

34. Yoshioka M, Crum MM, Sample JT: Autorepression of Epstein-Barr virus nuclear antigen 1 expression by inhibition of pre-mRNA processing J Virol 2008, 82:1679-1687.

35. Altmann M, Pich D, Ruiss R, Wang J, Sugden B, Hammerschmidt W: Transcriptional activation by EBV nuclear antigen 1 is essential for the expression of EBV's transforming genes. Proc Natl Acad Sci USA 2006, 103:14188-14193.

36. Nilsson T, Zetterberg $H$, Wang YC, Rymo L: Promoter-proximal regulatory elements involved in oriP-EBNA1-independent and -dependent activation of the Epstein-Barr virus C promoter in B-lymphoid cell lines. J Virol 2001, 75:5796-5811.

37. Borestrom C, Forsman A, Ruetschi U, Rymo L: E2F1, ARID3A/Bright and Oct-2 factors bind to the Epstein-Barr virus $C$ promoter, EBNA1 and oriP, participating in long-distance promoter-enhancer interactions. J Gen Virol 2012, 93:1065-1075.

38. Sompallae R, Callegari S, Kamranvar SA, Masucci MG: Transcription profiling of Epstein-Barr virus nuclear antigen (EBNA)-1 expressing cells suggests targeting of chromatin remodeling complexes. PLoS One 2010, 5:e12052.

39. Takacs M, Segesdi J, Banati F, Koroknai A, Wolf H, Niller HH, Minarovits J: The importance of epigenetic alterations in the development of epstein-barr virus-related lymphomas. Mediterr J Hematol Infect Dis 2009, 1:e2009012.

40. Paschos K, Allday MJ: Epigenetic reprogramming of host genes in viral and microbial pathogenesis. Trends Microbiol 2010, 18:439-447.

41. Sudo M, Chong JM, Sakuma K, Ushiku T, Uozaki H, Nagai H, Funata N, Matsumoto $\mathrm{Y}$, Fukayama M: Promoter hypermethylation of E-cadherin and its abnormal expression in Epstein-Barr virus-associated gastric carcinoma. International journal of cancer Journal international du cancer 2004, 109:194-199.

42. Sakuma K, Chong JM, Sudo M, Ushiku T, Inoue Y, Shibahara J, Uozaki H, Nagai H, Fukayama M: High-density methylation of p14ARF and p16INK4A in Epstein-Barr virus-associated gastric carcinoma. International journal of cancer Journal international du cancer 2004, 112:273-278.

43. Osawa T, Chong JM, Sudo M, Sakuma K, Uozaki H, Shibahara J, Nagai H, Funata N, Fukayama M: Reduced expression and promoter methylation of p16 gene in Epstein-Barr virus-associated gastric carcinoma. Jpn J Cancer Res 2002, 93:1195-1200.

44. Chong JM, Sakuma K, Sudo M, Ushiku T, Uozaki H, Shibahara J, Nagai H, Funata N, Taniguchi H, Aburatani H, Fukayama M: Global and non-random CpG-island methylation in gastric carcinoma associated with Epstein-Barr virus. Cancer Sci 2003, 94:76-80

45. Chang MS, Uozaki H, Chong JM, Ushiku T, Sakuma K, Ishikawa S, Hino R, Barua RR, Iwasaki Y, Arai $\mathrm{K}$, et al: $\mathrm{CpG}$ island methylation status in gastric carcinoma with and without infection of Epstein-Barr virus. Clin Cancer Res 2006, 12:2995-3002.

46. Chau CM, Zhang XY, McMahon SB, Lieberman PM: Regulation of EpsteinBarr virus latency type by the chromatin boundary factor CTCF. J Virol 2006, 80:5723-5732

47. Tempera I, Wiedmer A, Dheekollu J, Lieberman PM: CTCF prevents the epigenetic drift of EBV latency promoter Qp. PLoS Pathog 2010, 6(8):e1001048.

48. Imai S, Nishikawa J, Takada K: Cell-to-cell contact as an efficient mode of Epstein-Barr virus infection of diverse human epithelial cells. J Virol 1998, 72:4371-4378

49. Chau CM, Lieberman PM: Dynamic chromatin boundaries delineate a latency control region of Epstein-Barr virus. J Virol 2004, 78:12308-12319.

50. Lu F, Day L, Gao SJ, Lieberman PM: Acetylation of the latency-associated nuclear antigen regulates repression of Kaposi's sarcoma-associated herpesvirus lytic transcription. J Virol 2006, 80:5273-5282.

51. Lu F, Stedman W, Yousef M, Renne R, Lieberman PM: Epigenetic regulation of Kaposi's sarcoma-associated herpesvirus latency by virus-encoded microRNAs that target Rta and the cellular Rbl2-DNMT pathway. J Virol 2010, 84:2697-2706.

\section{doi:10.1186/1743-422X-11-12}

Cite this article as: Lu et al.: EBNA1 binding and epigenetic regulation of gastrokine tumor suppressor genes in gastric carcinoma cells. Virology Journal 2014 11:12. 